\title{
Collectivity of motion in undercooled liquids and amorphous solids
}

\author{
H. R. Schober \\ Institut für Festkörperforschung, Forschungszentrum Jülich, D-52425 Jülich, Germany
}

(October 26, 2018)

\begin{abstract}
The motion of the structure determining components is highly collective, both in amorphous solids and in undercooled liquids. This has been deduced from experimental low temperature data in the tunneling regime as well as from the vanishing isotope effect in diffusion in glasses and undercooled liquids. In molecular dynamics simulations of glasses one observes that both low frequency resonant vibrations and atomic jumps are centered on more than 10 atoms which, in densely packed materials, form chainlike structures. With increasing temperature the number of atoms jumping collectively increases. These chains of collectively jumping atoms are also seen in undercooled liquids. Collectivity only vanishes at higher temperatures. This collectivity is intimately related to the dynamic heterogeneity which causes a non-Gaussianity of the atomic displacements.
\end{abstract}

\section{INTRODUCTION}

Research into the physics of glasses spans a large temperature range, from the liquid to near $0 \mathrm{~K}$. There are a number of characteristic temperatures. The melting temperature, $T_{\mathrm{m}}$, marks the crossover to the undercooled regime, where the liquid is no longer the thermodynamic ground state. The Kauzmann temperature, $T_{\mathrm{K}}$, is the temperature where the entropy of the liquid would fall below the one of the corresponding crystal. It is sometimes interpreted as an ideal glass transition temperature. Before $T_{\mathrm{K}}$ is reached upon cooling, any real system arrests at the glass-transition temperature, $T_{\mathrm{g}}$, into a solid like state, the glass. This temperature $T_{\mathrm{g}}$ shows e.g. as a change in volume expansion or a (smeared) jump in the specific heat. By definition one has in all systems $T_{\mathrm{K}}<T_{\mathrm{g}}<T_{\mathrm{m}}$. The exact value of the glass-transition temperature depends on cooling rate, pressure and other parameters [1].

In many materials one observes a sharp upturn of the viscosity curves at temperatures well above $T_{\mathrm{g}}$. This change in the dynamics of the melt is taken in theories such as the mode coupling theory (MCT) [2] as the true transition which defines a temperature $T_{\mathrm{c}}>T_{\mathrm{g}}$.

At the lowest temperatures, below $\approx 1 \mathrm{~K}$, the dynamics in glasses is dominated by twolevel systems [3] which can be described by the tunneling model which was formulated nearly thirty years ago [4.5]. In addition to these tunneling states, one observes local relaxations in glasses and at frequencies near $1 \mathrm{THz}$ an excess of vibrations compared to the Debye spectrum, given by the sound waves. This excess leads to the "boson peak" in the inelastic 
scattering intensity. To describe the excess low energy excitations the tunneling model was extended to the soft potential model [6,7]. From this model "universal" temperature dependencies can be derived for temperatures of some $10 \mathrm{~K}$. Comparing the model to experiment one finds that the excess low energy excitations are collective motions of 10 to 100 atoms [8,9]. Similar values were found by computer simulations for the low frequency resonant modes [10].

The most popular model to describe the under-cooled liquid is the mode coupling theory [2]. In this statistical model a nonlinear memory kernel leads to a blocking of modes at $T_{\mathrm{c}}$. It predicts scaling relations and dependencies of the form $\left(T-T_{\mathrm{c}}\right)^{\gamma}$ for quantities such as the diffusion constant. Many aspects of this theory have been at least semi quantitatively verified by computer simulations, see e.g. [11].

At present there is no common theory for both the glassy and the super-cooled state. The transition from one regime into the other is probed by experiment and increasingly by computer simulations. The accumulated data should help to probe the limits of the existing theories and guide towards a theory bridging the present gap between glass and liquid theories.

In this contribution we will review computer simulations both in the glass and in the liquid. The emphasis will be on collective effects observed in both.

\section{SIMULATION MODELS}

Our molecular dynamics simulations are done for systems of 500 to 10000 atoms with periodic boundary conditions. The equations of motion are integrated by the velocity Verlet algorithm with a time-step of order fs. We use cooling rates of $\geq 10^{11} \mathrm{~K} / \mathrm{s}$ and aging times of order ns. For the model systems of soft spheres and Lennard-Jones atoms these values correspond to a conversion to Ar. Zero external pressure was exerted on all but the soft sphere systems where the volume was kept constant.

To check the material dependence of the results we use model systems (soft spheres (SSG) and Lennard-Jones (LJ), both monatomic and binary) and models of the binary metallic glass CuZr and of Se. Details of the simulations can be found in the references given further down.

The soft spheres (SSG) are described by a purely repulsive potential

$$
V(R)=\epsilon(\sigma / R)^{n}+V_{\text {cut }}
$$

where $n=6$ or $n=12$ and $V_{\text {cut }}$ is a small correction to give a smooth cutoff.

As a simple model potential which allows for zero external pressure we use a LennardJones (LJ) potential

$$
V(R)=4 \epsilon\left[(\sigma / R)^{12}-(\sigma / R)^{6}\right]+V_{\text {cut }} .
$$

We study both monatomic and binary LJ-systems. In the latter case we use the parameters of Kob and Anderson [11] with a different cutoff.

As a typical example of a binary metallic glass we simulate $\mathrm{Cu}_{33} \mathrm{Zr}_{67}$, described by a modified embedded atom potential. For details see [12]. The results do not strongly depend 
on the choice of interaction. As far as data are available there is good agreement with the similar NiZr system modeled with a totally different interaction

As an example of a good monatomic glass former we choose selenium. Se has a coordination number near 2. The amorphous structure consists of inter-netted rings and chains. We describe it by a potential of the Stillinger-Weber type. In this potential the covalent bonds are described by an additional three body term $V_{3}\left(\left|\mathbf{R}^{1}-\mathbf{R}^{2}\right|,\left|\mathbf{R}^{1}-\mathbf{R}^{3}\right|, \cos \Theta_{213}\right)$ where $\Theta_{213}$ is the apex angle [13]. The parameters were fitted to both molecular and crystalline data.

The examples presented in the following will be taken from simulations of these different materials. We will emphasize the qualitative aspects which are equal for all these systems.

\section{VIBRATIONS AND ATOMIC JUMPS IN THE GLASS}

The vibrations in a glass at low temperatures can be most easily studied in the harmonic approximation. As example we quench samples of up to 5488 atoms of a SSG to $T=0 \mathrm{~K}$. The atomic configuration will then correspond to a minimum of the potential energy. We can expand the energy in terms of the displacements from this minimum energy. The quadratic terms define a dynamical matrix

$$
D_{\alpha \beta}^{i j}=\frac{1}{\sqrt{m_{i} m_{j}}} \frac{\partial^{2} E_{p o t}(\{\mathbf{R}\})}{\partial R_{i, \alpha} \partial R_{j, \beta}},
$$

where $i, j$ denote the atoms and $\alpha, \beta$ the Cartesian coordinates. Diagonalization gives, in harmonic approximation, the frequencies of the eigenmodes of vibration and their eigenvectors, i.e. their spatial structure. Fig. 1 shows the vibrational spectrum, $Z(\nu)$, together with the corresponding Debye spectrum calculated from the elastic constants. The area between the two curves is due to the excess low frequency vibrations, typical for glassy structures. In a plot of $Z(\nu) / \nu^{2}$ one finds a maximum around $\nu=0.1$, the boson peak. The eigenmodes at the boson peak frequency have a complicated structure. At the lowest frequencies they can easily be identified as resonant (quasi-localized) vibrations. They can be decomposed into sound waves and soft local vibrations [14. The latter are in full accord with the predictions of the soft potential model [8]. The interaction with the local vibrations causes an attenuation of the sound waves. With increasing frequency the Ioffe-Regel limit is reached, the phonon mean free path drops to the wavelength. The increasing interaction between the modes leads to a level repulsion and to $Z(\nu) \propto \nu$. In the $Z(\nu) / \nu^{2}$ representation, or in the inelastic scattering intensity, this gives a drop $\propto \nu$ which causes the boson peak maximum.

It is important that the cores of the resonant vibrations are local but extend over many atoms. Any single atom is stable against isolated displacements. The atoms in the cores of resonant modes participate also in high frequency localized vibrations. This is typical for resonant modes caused by stresses due to configurational "defects". They are not due to some atoms weakly coupled to the rest.

The soft modes in glasses originate from some atomic configurations where some direction in the $3 N$-dimensional configuration space is locally soft. This softness should not be confused with the one of the sound-waves which are soft because all atoms move in phase. In the extreme case, the group of atoms forming the center of the soft quasi-local vibration 
is stabilized by the embedding matrix of the rest of the glass in a position lying between minima of the potential energy given by its near neighbors. To illustrate this, the dashed line in Fig. 2 shows the average potential energy of the 61 atoms which are most active in the given mode, $\langle U(x)\rangle_{\text {core }}$. Atoms are considered as active in a given mode if their amplitude, $\left|\mathbf{e}^{n}\right|$, is at least $30 \%$ of the maximal atomic amplitude in the mode. The partial potential energy of these active atoms is indeed double-well shaped with minima at $x_{\mathrm{m}} \approx \pm 1.3$, which corresponds to maximal displacements of individual atoms by $\left|\mathbf{R}^{n}-\mathbf{R}_{0}^{n}\right| \approx 0.2-0.3 \sigma$ from the equilibrium configuration. This maximal atomic displacement is of the order of the one observed in local low temperature relaxations [16].

At finite temperatures one observes these as aperiodic transitions from one local configurational minimum into another. To visualize these we monitor the total displacement $\Delta R$ from a starting configuration and define

$$
\Delta R(t)=\sqrt{\sum_{n}\left(\mathbf{R}^{n}(t)-\mathbf{R}^{n}(0)\right)^{2}}
$$

where $\mathbf{R}^{n}(t)$ is the position vector of particle $n$ at time $t$ and $\mathbf{R}^{n}(0)$ is the one at the starting or reference configuration. $\Delta R(t)$ oscillates due to the vibrations and changes due to relaxations, i.e. due to the transitions from one local energy minimum to another. An example is shown in Fig. 3 for the SSG at two temperatures. At $T=0.02 T_{\mathrm{g}}$ the glass clearly jumps between three configurations. Let us denote the configurations $A, B, C$. The jump sequence is $B \rightarrow C \rightarrow B \rightarrow C \rightarrow A \rightarrow C \rightarrow A$. By quenching to $T=0 \mathrm{~K}$ we find the potential energy differences $\Delta E^{B}-\Delta E^{A}=1.74 \times 10^{-3} \epsilon$ and $\Delta E^{C}-\Delta E^{A}=1.81 \times 10^{-3} \epsilon$. These energy differences are of the order of the temperature. The corresponding spatial distances between the configurations, Eq. (四), are $\Delta R^{A-B}=1.63 \sigma, \Delta R^{A-C}=0.96 \sigma$, and $\Delta R^{B-C}=1.0 \sigma$, i.e. they are of the order of the nearest neighbor distance $R_{N N}=1.1 \sigma$. The maximal distance an individual atom travels in these jumps is only $0.3 \sigma$, about a quarter of $R_{N N}$. Such relaxations can be observed experimentally e.g. as telegraph noise in the electric resistivity of point contacts [17]. Increasing the temperature by a factor of four the average displacement in the initial configuration doubles as expected for a vibrational mean square displacement. The jumps seen at the lower temperature can no longer be resolved and new jumps over larger distances are observed.

All observed relaxations are collective jumps localized to 10 or more atoms forming twisted chain-like structures with some side branching [16]. An example of such a structure is shown in Fig. 4 [18]. The chain structure is a consequence of the dominance of the nearest neighbour bond in close packed structures. A low temperature relaxation is only possible if these bonds are not strongly compressed. Using the squared atomic displacement as "mass" we find a gyration radius of about 5 nearest neighbour distances for the chain of jumping atoms. The effective dimension is about 2 as one would expect for a twisted chain with side-branching. We observe similar structures in amorphous Se [19, 17] and CuZr [12,20].

In materials with a different inherent structure these relaxations will be different. In $\mathrm{SiO}_{2}$ we would expect them to be collective twists of tetrahedra as has been postulated for the soft vibrations [21]. The origin will be, however, the same, namely local stresses leading to a softness in one direction of the multidimensional configuration space.

Increasing the temperature we observe a marked increase of the number of atoms participating in a single jump [22]. 
The local relaxations show two important correlations. First, in accord with the soft potential model they are strongly correlated with the soft vibrations [19, 16]. Secondly, there is also a strong but not full correlation between subsequent jumps in the same part of the sample. This latter correlation indicates that one jump can trigger another one which leads to a correlation in time, i. e. bursts of jumps [23]. It also leads to a slow increase in time of the number of atoms which have moved significantly, the dynamic heterogeneity. We illustrate it in Fig. 5 where we show the mobile atoms at $T \approx 0.15 T_{g}$ after some ns (in Ar units). The effect is again independent of the specific material studied, whereas the size of the region at a given time and temperature will depend on the particulars of the inter-atomic interaction. The atoms which have moved in a given time interval form a complicated structure. From the time dependence of a suitably defined correlation function a fractal dimension has been derived for a binary soft sphere glass [24]. The time dependence of the dynamic heterogeneity is reflected also in the time dependence of the non-Gaussianity discussed further down.

\section{SIMULATIONS IN THE LIQUID}

Computer simulations in the liquid state, especially with respect to MCT, have been discussed in numerous papers and reviews, see e.g. [11,25,226]. The predictions of MCT are at least semi-quantitatively reproduced in these calculations. We have not done extensive tests. However, quantities such as the intermediate self-scattering function and the self diffusion constant follow the trends of MCT for both our super-cooled CuZr [27] and Se [28]. Tests of MCT are widely discussed elsewhere in this conference.

In the liquid state it is no longer straightforward to separate single jumps. To obtain information on the atomic structure of the motion one can study the difference between structures some ps apart. To remove the effects of vibrations these configurations have to be averaged over a typical vibrational period. Fig. 6 shows an example for under-cooled liquid $\mathrm{Cu}_{33} \mathrm{Zr}_{67}$. There are two striking effects. First, clearly the smaller $\mathrm{Cu}$ atoms are much more mobile. Secondly we observe string like structures similar to the ones shown for the amorphous materials, Figs. 4 and 5. These structures indicate a high degree of collectivity in the motion also in the under-cooled liquid. They have been studied extensively for a binary Lennard-Jones system by Donati et al. [29]. These authors report a marked increase of collectivity when the liquid is quenched to $T_{\mathrm{c}}$. These mobile strings again will lead to a dynamic heterogeneity. If one marks the atoms which have moved in a given time intervall by further than some cutoff distance, one observes structures similar to the one shown in Fig. 5 for the glassy state [30]. Experimental evidence for collective particle motion was gained by neutron scattering [31] its conclusiveness was, however, disputed [32]. Additional evidence is again the vanishing isotope effect in diffusion [33].

\section{ISOTOPE EFFECT IN DIFFUSION}

The isotope effect is the most direct experimental probe of collectivity. In a monatomic liquid the diffusion constant can be written 34]

$$
D=D_{0} f(T, \rho)=D_{0}^{*} f(T, \rho) / \sqrt{m}
$$


where $T$ is the temperature, $\rho$ the atomic density and $m$ is the mass of the diffusing particle. In the case of different components and different isotopes, considered here, the situation is more complicated. At low densities and high temperatures when diffusion is dominated by binary collisions the kinetic approximation should hold and Eq. 5 should apply approximately for each constituent. Lowering the temperature or increasing the density effects of collective motion will gain importance.

A frequently used measure of this effect is the isotope effect parameter $E$ [35]

$$
E_{\alpha \beta}^{\ell}=\frac{D_{\alpha}^{\ell} / D_{\beta}^{\ell}-1}{\sqrt{m_{\beta}^{\ell} / m_{\alpha}^{\ell}}-1}
$$

where the index $\ell$ denotes the different chemical components and $\alpha$ and $\beta$ denote different isotopes. For a tracer atom with a mass of $\bar{m}^{\ell}+\delta m^{\ell}$ (average mass $\bar{m}^{\ell}$ ) the change of the diffusion constant is then in linear approximation [36]

$$
\frac{\Delta D^{\ell}}{\overline{D^{\ell}}}=-\frac{\delta m^{\ell}}{2 \bar{m}^{\ell}} E
$$

An isotope effect parameter of $E_{\alpha \beta}^{\ell} \approx 1$ indicates uncorrelated single particle motion whereas collectivity results in $E_{\alpha \beta}^{\ell} \rightarrow 0$.

Formally one can describe the isotope effect by an effective mass

$$
\left(m_{\alpha}^{\ell}\right)_{\mathrm{eff}}=m_{\alpha}^{\ell}+\left(N_{D}^{\ell}-1\right) \bar{m}
$$

where $N_{D}$ stands for the effective number of particles moving cooperatively. Such a definition can be justified well in the solid state. There, one has well defined diffusional jumps and $N_{D}$ is defined by the multidimensional jump vector. Here we introduce $N_{D}$ only formally. The mass dependence of the diffusion constant is then given by

$$
D_{\alpha}^{\ell}=f^{\ell}(T, \rho) / \sqrt{\left(m_{\alpha}^{\ell}\right)_{\mathrm{eff}}} .
$$

From Eq. 6 one gets then $E_{\alpha \beta}^{\ell} \approx 1 / N_{D}^{\ell}$.

Evidence from earlier measurements of the isotope effect [34 is conflicting. Progress was made by simultaneously measuring the diffusion of the tracer atoms ${ }^{57} \mathrm{Co}$ and ${ }^{60} \mathrm{Co}$ [37]. Using this technique for diffusion of $\mathrm{Co}$ in amorphous $\mathrm{Co}_{76.7} \mathrm{Fe}_{2} \mathrm{Nb}_{14.3} \mathrm{~B}_{7}$ a value $E=0.1$ was found indicating a high degree of collectivity. In contrast for self diffusion in crystalline Co one finds $E=0.7$. There, diffusion is by a vacancy mechanism which involves essentially single particle jumps with not too large displacements of the neighbors. The technique was also applied to a super-cooled melt of $\mathrm{Zr}_{46.7} \mathrm{Ti}_{8.3} \mathrm{Cu}_{7.5} \mathrm{Ni}_{10} \mathrm{Be}_{27.5}$, and again a very low isotope effect was observed [33].

We have calculated the isotope effect for a monatomic [38] and binary [39] Lennard-Jones liquids. Fig. 7 shows the isotope effect parameters for both components of a binary LJ-liquid, consisting of $80 \%$ large and $20 \%$ small atoms as used in many other simulations, see e.g. [11]. We find that the values are relatively low in the whole temperature range investigated, except for the high temperature ones of the smaller component. The behaviour for the larger atoms closely resembles the one observed in the mono-atomic LJ-system. These atoms form the backbone of the structure. The values for the smaller atoms show the same decrease 
with temperature but are always clearly higher than those of the larger atoms. This reflects their smaller size. At the highest temperatures the small atoms seem to move through the matrix of larger atoms by binary collisions only. The observed high collectivity near $T_{c}$ is in agreement with the experimental findings of Ehmler et al. 33. who found $E \approx 0.09$ for Co diffusion in super-cooled liquid $\mathrm{Zr}_{46.7} \mathrm{Ti}_{8.3} \mathrm{Cu}_{7.5} \mathrm{Ni}_{10} \mathrm{Be}_{27.5}$. It is similar to the one found in the monatomic soft sphere glass at low temperatures [16]. The Decrease of the isotope effect with lower temperatures is proportional to the increase in density. The proportionality factors are, however, different for the two components. The effect of the density on the diffusion constant and on $E$ are different, the dynamics clearly depends on density and temperature.

The high collectivity both above and below the glass transition is related to the dynamic heterogeneity which we expect, therefore, to behave similarly. An experimentally accessible quantity which probes heterogeneity is the non-Gaussianity which we will discuss in the last section.

\section{DYNAMIC HETEROGENEITY}

The change of dynamics upon cooling the melt towards the glass transition can clearly be seen in the self part of the van Hove function $G^{s}(r, t)$ which is related to the probability that an atom has moved by a distance $r$ during a time $t$ :

$$
P(r, t)=4 \pi r^{2} G^{s}(r, t)=\left\langle\delta\left(r-\left|\mathbf{R}^{n}(t)-\mathbf{R}^{n}(0)\right|\right)\right\rangle .
$$

At high temperatures $P(r, t)$ is, apart from the geometrical factor $4 \pi r^{2}$ nearly perfectly Gaussian and broadens $\propto \sqrt{t}$. Upon cooling towards $T_{c}$, and beyond, a tail to larger $r$-values grows with time. The self correlation function becomes markedly non-Gaussian and a tail to larger distances grows with time. Finally approaching $T_{c}$ additional structure evolves, particularly for the more mobile components, [40,41,11, 42,27]. As example Fig. 8 shows the distribution of the atomic displacements of $\mathrm{Zr}_{67} \mathrm{Cu}_{33}$ at $T=1000 \mathrm{~K}$ after a time $t=210 \mathrm{ps}$, i.e. during the early time of the so called $\alpha$-relaxation. The second peak grows at a fixed, time independent, position roughly equal to the mean nearest neighbor distance. From this structure of $P(r, t)$ one can conclude that there are preferred positions on the relevant time scales. This does, however, not necessarily mean that these positions are reached in a single jump, as frequently assumed. From an analysis of the evolution with time it has been concluded that the evolution of this structure in $P(r, t)$ is accompanied with a strong increase of back correlation [43].

This strong deviation of $P(r, t)$ indicates that some atoms have been much more mobile than the average. This effect is called dynamic heterogeneity. To quantify it the nonGaussianity parameter 44] is often used

$$
\alpha_{2}(t)=\frac{3<\Delta r^{4}(t)>}{5<\Delta r^{2}(t)>^{2}}-1
$$

where $\langle\ldots\rangle$ denotes time averaging, $\Delta r^{2}(t)$ is the mean square displacement and $\Delta r^{4}(t)$ is the mean quartic displacement. This parameter is defined so that it is equal to zero when the atomic motion is homogeneous. Experimentally it can be obtained from the $q$-dependence of the Debye-Waller factor 45]. It has also been calculated in numerous molecular dynamics 
simulations of liquids, e.g. by Kob et al. for the binary Lennard-Jones system [11,46]. The limiting values for both times $t=0$ and $t=\infty$ is $\alpha_{2}=0$. The latter limit reflects the ergodicity of the system for long times. Starting from $t=0, \alpha_{2}(t)$ rises in general monotonically to a maximum from where it drops again monotonically. The maximum value is around 0.2 in the hot liquid and rises strongly in the under-cooled liquid, where a maximum value of 3 has been reported [11]. The position of the maximum in time is at high temperatures in the ps range and correlates in the super-cooled liquid with the onset of the $\alpha$-relaxation which is attributed to long range motion. This general behavior is observed in all systems we have studied.

In the previous sections we have seen that on an atomic level the dynamics in the glass and the under-cooled liquids are very similar. To see this quantitatively for the heterogeneity we have calculated $\alpha_{2}(t)$ for different temperatures above and below the glass transition. In Fig. 8 we show this for both components of the binary LJ-system in a log-log representation of $t \cdot \alpha_{2}(t)$ versus time. First we see the general trends discussed above. These hold not only in the liquid but also in the glass. Secondly we observe an asymptotic linear increase

of $\alpha_{2}(t) \propto \sqrt{t}$. For the lower temperatures this behaviour stretches over several ns (when one converts the LJ-units to ones appropriate for Ar). This dependence can be explained by the above mentioned strong correlation between successive collective jumps [47]. One can envisage a chain of atoms jumping. This jump can either be reversed in a successive jump or might trigger a jump of another chain which will involve many but not all atoms which have jumped previously.

\section{CONCLUSION}

We have shown by computer simulations of different materials that motion both in the glass and in the under-cooled liquid is highly collective. The dynamics in the glass at low temperatures can be described by the soft potential model which postulates similar structures for tunneling centers, quasi localized vibrations and local relaxations. These local motions involve groups of ten and more atoms forming in dense packed systems predominantly chain structures. These structures are closely related to resonant vibrations which cause the boson peak in the inelastic scattering intensity. Successive jumps in the same part of the sample are correlated. With increasing temperature the number of atoms jumping collectively increases in the amorphous state. Similar collectivity is also observed in the undercooled liquid where it increases upon quenching. The decrease of the isotope effect of the diffusion upon quenching to the glass transition indicates again an increase in collectivity, in agreement with experiment and other simulations. The collectivity of motion is related to the dynamic heterogeneity. The non-Gaussianity parameter, a measure of the dynamic heterogeneity, increases rapidly in the undercooled liquid and varies smoothly through the glass transition.

\section{ACKNOWLEDGMENT}

The author acknowledges the essential contributions by his coworkers B. B. Laird, C. Oligschleger, C. Gaukel, M. Kluge, D. Caprion and V. L. Luchnikov. This work was par- 
tially supported by the Deutsche Forschungsgemeinschaft in the Schwerpunkt "Unterkühlte Metallschmelzen: Phasenselektion und Glasbildung". We also acknowledge the financial support by the A. von Humboldt foundation. 


\section{REFERENCES}

[1] P. R. Okamoto, N. Q. Lam, and L. E. Rehn, Solid State Physics, edited by H. Ehrenreich and F. Saepen (Academic Press, San Diego, 1999), Vol. 52, p. 2.

[2] W. Götze and A. Sjölander, Rep. Prog. Phys 55, (1992) 241.

[3] Amorphous Solids: Low Temperature Properties, edited by W. A. Phillips (SpringerVerlag, Berlin, 1981).

[4] W. Phillips, J. Low Temp. Phys. 7, (1972) 351.

[5] P. W. Anderson, B. I. Halperin, and C. M. Varma, Philos. Mag. 25, (1972) 1.

[6] V. G. Karpov, M. I. Klinger, and F. N. Ignatiev, Sov. Phys. JETP 57, (1983) 439.

[7] M. A. Il'in, V. G. Karpov, and D. A. Parshin, Sov. Phys. JETP 65, (1983) 165.

[8] U. Buchenau, Y. M. Galperin, V. L. Gurevich, and H. R. Schober, Phys. Rev. B 44, (1991) 5093.

[9] U. Buchenau et al., Phys. Rev. B 46, (1992) 2798.

[10] B. B. Laird and H. R. Schober, Phys. Rev. Lett 66, (1991) 636; H.R. Schober and B. B. Laird, Phys. Rev. B 44 (1991) 6746.

[11] W. Kob and H. Andersen, Phys. Rev. E 51, (1995) 4626; ibid. 52, (1995) 4134.

[12] C. Gaukel, Berichte des Forschungszentrums Jülich 3556, (1998).

[13] C. Oligschleger, R. O. Jones, S. M. Reimann, and H. R. Schober, Phys. Rev. B 53, 6165 (1996).

[14] H. R. Schober and C. Oligschleger, Phys. Rev. B 53, 11469 (1996).

[15] V. A. Luchnikov, N. N. Medvedev, Yu. I. Naberukhin and H. R. Schober, Phys. Rev. B 62 (2000) 3181.

[16] C. Oligschleger and H. R. Schober, Phys. Rev. B 59, 811 (1999).

[17] V. I. Kozub and C. Oligschleger, J. Phys.: Condens. Matter 10, 8033 (1998).

[18] H. R. Schober, C. Oligschleger, and B. B. Laird, J. Non-Cryst. Sol. 156, 965 (1993).

[19] C. Oligschleger and H. R. Schober, Solid. State Commun. 93, 1031 (1995).

[20] C. Oligschleger, C. Gaukel, and H. R. Schober, J. Non-Cryst. Sol. 250-252, 660 (1999).

[21] U. Buchenau, N. Nücker, and A. J. Dianoux, Phys. Rev. Lett. 53, 2316 (1984).

[22] H. R. Schober, C. Gaukel, and C. Oligschleger, Defect and Diffusion Forum 143-147, 723 (1997).

[23] H. Teichler, J. Non-Cryst. Solids in print.

[24] G. Parisi, J. Phys. Chem. B 103, 4128 (1999).

[25] H. Teichler, Phys. Rev. Lett. 76, 62 (1996).

[26] W. Kob, J. Phys.: Condens Matter 11, R85 (1999).

[27] C. Gaukel, M. Kluge, and H. R. Schober, J. Non-Cryst. Sol. 250-252, 664 (1999).

[28] D. Caprion and H. R. Schober, Phys. Rev. B 62 (2000) 3709.

[29] C. Donati et al., Phys. Rev. Lett. 80, 2338 (1998).

[30] T. Muranaka and Y. Hiwatari, J. Phys. Soc. Japan 67, 1982 (1998).

[31] M. Russina, F. Mezei, R. Lechner, S. Longeville, and B. Urban, Phys. Rev. Lett 84, 3630 (2000).

[32] W. Schmidt, M. Ohl, and U. Buchenau, Phys. Rev. Lett. 85, 5669 (2000); M. Russina et al. ibid p. 5670 .

[33] H. Ehmler et al., Phys. Rev. Lett. 80, 4919 (1998).

[34] H. J. V. Tyrrell and K. R. Harris, Diffusion in Liquids (Butterworth, London, 1984).

[35] A. H. Schoen, Phys. Rev. Lett. 1, 138 (1958). 
[36] F. Lantelme, P. Turq, and P. Schofield, J. Chem. Phys 67 (1977) 3869.

[37] F. Faupel, P. W. Hüppe, and K. Rätzke, Phys. Rev. Lett. 65, 1219 (1990).

[38] M. Kluge and H. R. Schober, Phys. Rev. E in print (2000).

[39] H. R. Schober, Solid State Commun. 119 (2001) 73.

[40] J. N. Roux, J. L. Barrat, and J. P. Hansen, Phys. Rev. A 38 (1989) 454.

[41] G. Wahnstrøm, Phys. Rev. A 44 (1991) 3752.

[42] H. Teichler, Defect and Diffusion Forum 143-147 (1997) 717.

[43] C. Gaukel and H. R. Schober, Solid State Commun. 107 (1998) 1.

[44] A. Rahman, Phys. Rev. 136, A405 (1964).

[45] R. Zorn, Phys. Rev. B 55, 6249 (1997).

[46] W. Kob et al., Phys. Rev. Lett. 79, 2827 (1997).

[47] D. Caprion, J. Matsui, and H. R. Schober, Phys. Rev. Lett 85 (2000) 4293. 


\section{FIGURES}

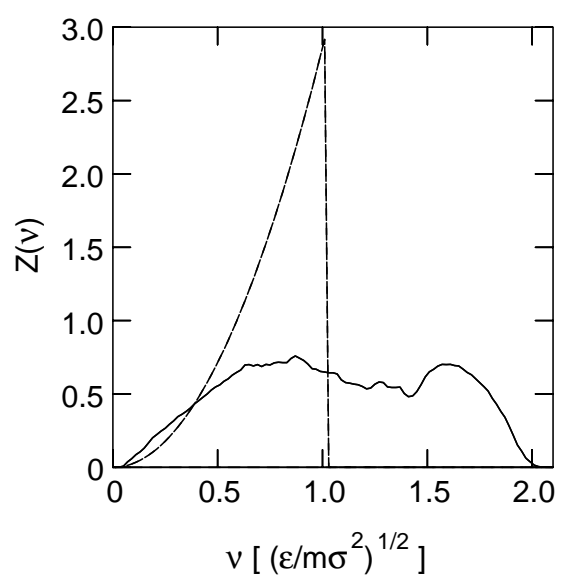

FIG. 1. Configurationally averaged vibrational density of states of the soft sphere glass (solid line) with $n=6$ and Debye spectrum (dashed line) [14]

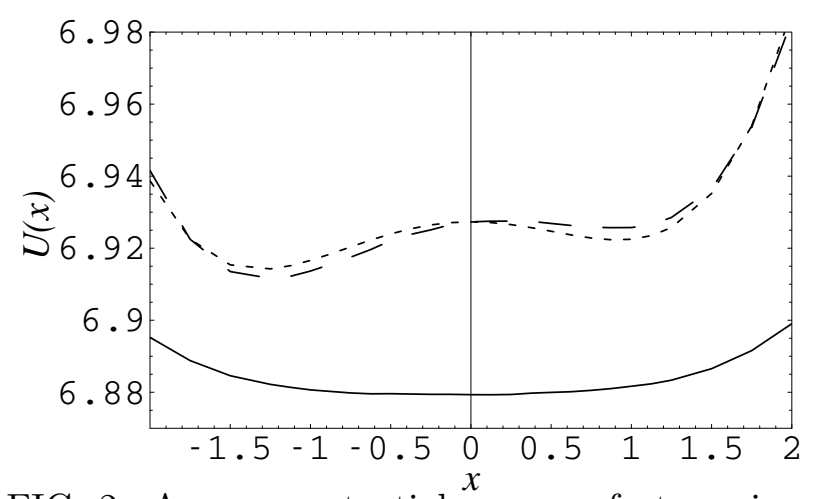

FIG. 2. Average potential energy of atoms in a single soft vibrational mode with frequency $\nu=0.0985$, participation ratio $p=0.23$. Solid line: potential energy averaged over all atoms in the system. Dashed line: partial potential energy, averaged over 61 atoms of the core of the mode. Dotted line: least squares fit of the partial potential energy by a soft potential polynomial [15]. The average nearest neighbour distance is $1.1 \sigma$. 


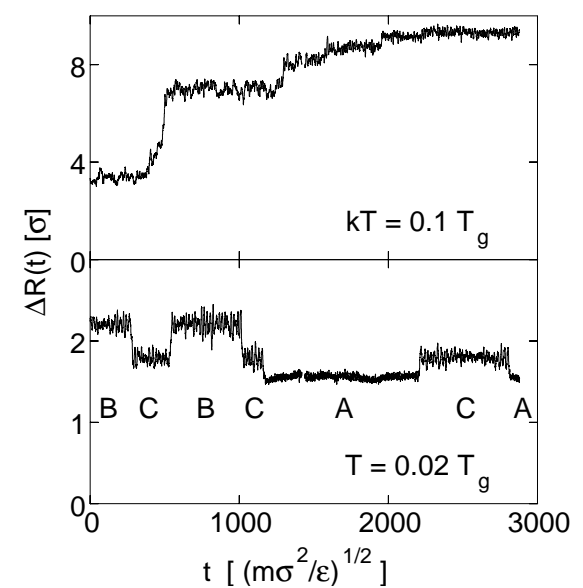

FIG. 3. Total displacement relative to a local minimum configuration as function of time for one sample of the glass of Fig. 1 with $N=5488$ at two temperatures. Please note the different scales for $\Delta R$ 16.

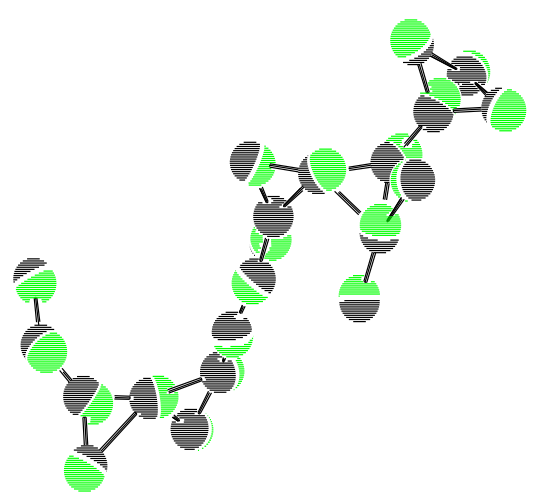

FIG. 4. Collective jump in the glass of Fig. 1 at $T=0.15 T_{\mathrm{g}}$. The initial positions of the atoms are shown by the dark spheres and the final ones by the shaded spheres. The bonds connect nearest neighbours. Shown are all atoms with more than $40 \%$ of the maximal atomic displacement [18. 


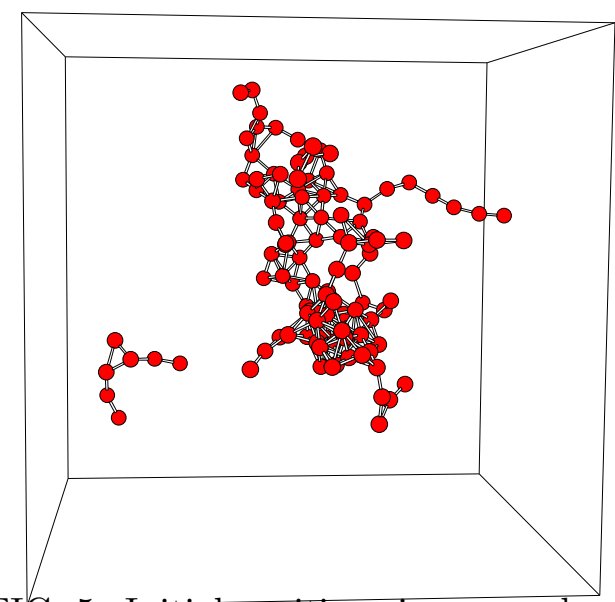

FIG. 5. Initial positions in a sample of the glasses of atoms displaced by more than 0.3 of the average nearest neighbour distance during a time interval $\Delta t=3600\left(m \sigma^{2} / \epsilon\right)^{1 / 2}$ at $T \approx 0.15 T_{\mathrm{g}}$. The total displacement is approximately 8 nearest neighbour distances [16].

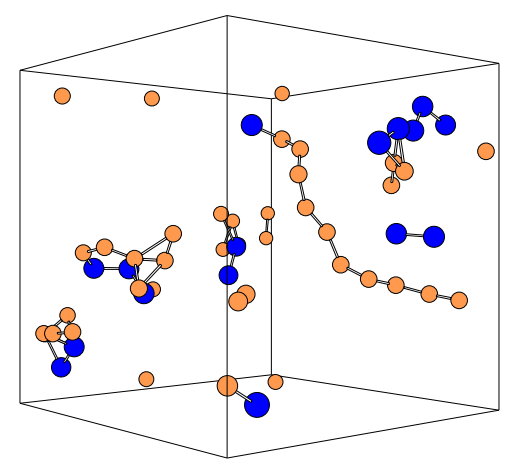

FIG. 6. Atoms in a melt of $\mathrm{Cu}_{33} \mathrm{Zr}_{67}$ at $T=1200 \mathrm{~K}$ with the largest displacements between time averaged configurations separated by 6.5 ps. Shown are $\mathrm{Cu}$ (light spheres) and $\mathrm{Zr}$ (dark spheres) atoms displaced by more than $1.6 \AA$ and $1.45 \AA$, respectively [22. 


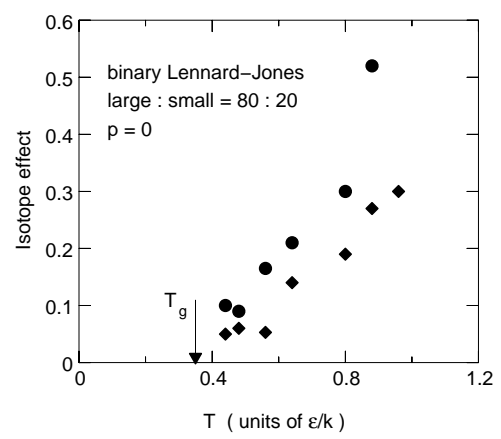

FIG. 7. Isotope effect in a binary LS-liquid as function of temperature at equilibrium density. The diamonds and circles refer to the larger and smaller atoms, respectively. The arrow indicates the glass transition temperature [39].

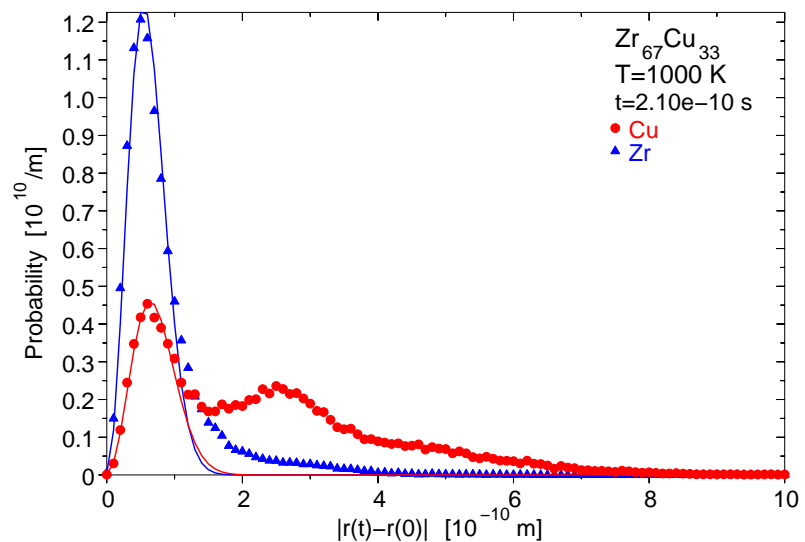

FIG. 8. Distribution of the atomic displacements $P(r, t)$ in $\mathrm{Cu}_{33} \mathrm{Zr}_{67}$ near $T_{c}$ after $210 \mathrm{ps,}$ calculated in MD (symbols). The line shows a Gauss-fit to the small distance part [12]. 


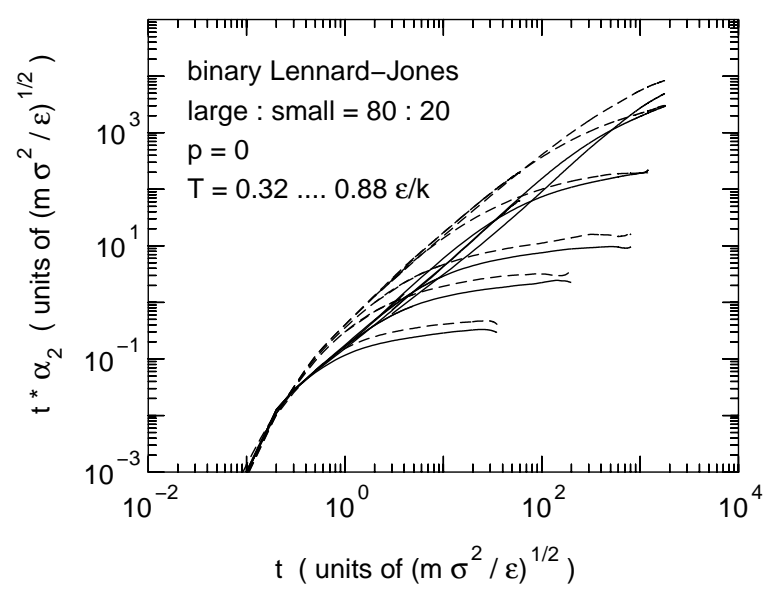

FIG. 9. Non-Gaussianity parameter multiplied by time for the binary LJ-system of Fig. 7 above and below the glass transition temperature. The solid and dashed lines refer to the larger majority and smaller minority components, respectively. The curves refer to the temperatures (from bottom to top): $0.88,0.56,0.48,0.40,0.36$ and 0.32 in units of $\epsilon / k$. 